\title{
Beiträge zur Naturgeschichte der Schmetterlinge
}

von

Anton Schmid in Frankfurt a. M.

\section{Larentia Aquilaria H.-S.}

D

ie Raupe sehr sparsam zur Endhälfte September erwachsen auf dem Augentrost (Euphrasia lutea), dessen Samen sie verzehrt. Sie erreicht eine Grölse von 6 Linien; ihr Körper ist kurz, gedrungen, nur nach vorn etwas gestreckt, lederbraun, seitlich heller verwaschen, weiter unten mit scharfer dunkelbrauner Abgränzung, die sich bis zur Bauchmitte vollends verliert.

Der kleine Kopf glänzend, hellgrundig mit dunkeln Atomen; vom gleichfarbigen Nacken bis zur Schwanzspitze ziehen 5 braune, oft nur schwach angedeutete Linien, wovon die mittleren zuletzt in einem braunen Winkel aufgehen.

Schwarze Punkte mit kurzen Härchen auf heller Basis überall einzeln zerstreut; die Krallen glänzend und gleich den Schwanzfüssen schwarz gefleckt.

Verwandlung in der Erde.

Der Spanner entwickelt sich Ende Juli und Anfangs August, zuweilen auch nach einer zweijährigen Puppenruhe.

Auf der Mombacher Haide bei Mainz.

\section{Botys Sanguinalis $\mathbf{L}$.}

Die Raupe (den 28. Juli 1861 entwickelt) lebt von den Blüthen des Thymus serpillum, indem sie sich von diesen röhrenartige Gänge bildet. Sie erreicht ausgewachsen eine Länge von 5 Linien.

Ihr Körper, in seiner äufseren Form an Pterophoriden-Raupen erinnernd, ist von rothbrauner, auch grünlicher Färbung und trägt über dem Rücken stets eine sehr wahrnehmbare fast weifse Linie. 
Der Kopf ist klein, glänzend, hellbraun, seitlich schwarz punktirt; die Frefsspitzen sind dunkel gerandet. Das Nackenschild ist gefleckt, nach hinten zu, neben dem hellen Rückenstreifen mit einer doppelten Reihe heller Wärzchen, mit braunen kurzen Borsten auf schwarzem Grunde versehen. Die untere Seite des Körpers mit den Krallen und Bauchfülsen ist schmutziggelb.

Die Verwandlung geschieht in einem länglichen, starken, mit Erdkörnern vermengten Gespinnste. Spätlinge der Raupen überwintern darin.

Der Zünsler vom Mai bis Seplember auf trockenen Sandstellèn mit magerer Vegetation.

Um Frankfurt am Hartweg; um Mombach und Budenheim bei Mainz; um Eberstadt bei Darmstadt.

\section{Nephopteryx Albicilla H.-S.}

Die Raupe lebt Ende August und Anfang September an Salweiden (Salix caprea) in leichten Gespinnsten zwischen Blättern. Sie erreicht eine Länge von 7 Linien.

Sie ist durchaus schön saftgrün; Kopf und Nackenschild glänzend mit dunkeln Atomen. Ueber den Rücken ziehen $\mathbf{5}$ grünliche Linien bis zum After; die $\mathbf{Z}$ wischenfelder sind mit kleinen geschwungenen Strichen versehen. Einzelne schwarze Punkle sind, als Basis langer gelber Härchen, über den ganzen Körper zerstreut.

Die Ringeinschnitte sind von gelber Färbung.

Die Motte im Mai, selten.

Im Scheèrwald bei Frankfurt gefunden.

\section{Conchylis Zebrana Hübn.}

Die Raupe fand ich 1846 in röhrenartigen Gängen zwischen den Blüthen von Gnaphalium arenarium. Gröfse 4 Linien.

Durch die tiefen Ringeinschnitte hat der Körper ein runzeliges Aussehen. Sie ist bleichgelb; auf der Oberseile des 6ten Ringes befinden sich zwei gelbbraune Makeln, der Ausgang des Darmkanals scheint vor dem After als dunkelgrauer Fleck durch.

Der kleine Kopf ist glänzend, dunkel schwarzbraun, das Nackenschild von etwas hellerer Färbung, ebenso die Krallen. Auf der Schwanzspitze sind feine helle Härchen bemerkbar. Die Raupe wird, nachdem sie überwintert, im Frühjahr unter Pflanzenabfällen zu einer kurzen, glänzend-hellbraunen Puppe.

Der Wickler findet sich von der Endhälfte April bis Mitte Mai auf mageren Sandstellen, nicht häufig. 
Um Frankfurt auf der Bieberer Höhe und auf der Mombacher Haide bei Mainz gefunden.

\section{Tinea Corticella Curtis.}

Die Raupe wird bis zu 2 Linien lang, ist beinfarben, der Kopf glänzend, kastanienbraun, seitlich mit kurzen hellen Härchen versehen; die Frefswerkzeuge sind dunkel gerandet. Auf dem ersten Leibesringe stehen ein Paar schwarze, vorwärts gekrümmte Querstriche und hinter diesen zwei braune ausgebreitele Flecke.

Der Darmkanal stellenweise dunkel durchscheinend und Krallen und Bauchfüfse von der Körperfarbe nicht verschieden.

Die Puppe glänzend, hellrothbraun, von lebhafterer Färbung auf dem Rücken.

Der Schmetterling wurde mit den Raupen schon Mitte Mai und den ganzen Juni - Nachzügler noch im Juli - gefunden, jedoch im Freien wohl selten.

Die Raupe wurde von mir, einigemal schon in Unzahl, aus weifsen Pilzen, die an dem Stamme einer Weifsbuche wuchsen, auch mit faulem Holz Ende April oder Mitle Mai eingetragen.

\section{Tinea Parasitella Hübn.}

Aus Buchen- und Weidenschwämmen entwickelt sich der Schmetterling manchmal in Anzahl.

Die Raupe ist 6 Linien lang, schmutzig blassgelb.

Der Kopf ist herzförmig, glänzend gelbbraun; das Nackenschild dunkelbraun; der Darmkanal auf dem Rücken rothbraun durchschimmernd; oben auf jedem Ringe mit vier - seitwärts mit einem braunen Wärzchen versehen, als Basis feiner, kurzer, heller Härchen.

Die Motte nicht häufig Ende Mai und im Juni.

Bei Frankfurt in der Pracht im Stadtwald, auch in Gärten gefunden.

\section{Nemophora Swammerdammella L.}

Den von der Raupe bewohnten, im Frühling unter Laub vorkommenden, ziemlich flachen Sack bemerkte ich zuerst den 24. März 1856. Derselbe, aus mehreren halbmondförmigen, ausgeschnittenen Blattsı̈̈cken zusammengefügt, hat ein flaschenförmiges Aussehen, welches sich jedoch im zweiten Jahre verliert und alsdann nimmt derselbe eine länglich gerundete Gestalt an.

Die Raupe minirt in ihrer Jugend die Blätter von Eichen und Buchen. Später scheint sie von zarten Pflanzen, dürrem Laub und 
wohl auch von nackten Insekten zu leben. Nicht selten kann man die Raupe bald am vorderen, bald am hinteren Ende des Gehäuses wahrnehmen

Die Raupe selbst erreicht eine Gröfse von $3 \frac{1}{2}$ Linien. Die Körperfarbe ist graulich, vom glänzend schwarzen Kopf bis zum letzten Brustring mehr fleischfarben; das Nackenschild dunkelbraun, glänzend; die beiden Brustringe von kastanienbrauner Färbung.

Der Darmkanal stark durchscheinend, die Afterringe dunkelbraun.

Die Krallen schwarz, seitlich mit je drei dunkelbraunen Borstenwärzchen versehen. Zur Verwandlung wird der Sack mit einigen starken Fäden an Laub etc. befestigt. Die hellbraune Puppe mit weit überragenden Fühlerscheiden, die um das Schwanzende gewunden sind, tritt zur Entwicklung aus dem Sack bis über die Flügelscheiden hervor.

Die Motte ist sebr verbreitet und gemein Ende April und im Mai in Wäldern, um Himbeeren, Brombeeren, Salweiden etc.

\section{Adela Rufimitrella Scop.}

Die sacktragende Raupe entdeckte ich den 29. Oktober 1859 in der Nähe von Erysimum alliaria unter abgefallenem Laub, wovon sie sich, sowie von sonstiger keimender Vegetation, den Winter über zu ernähren schien. In ihrer Jugend lebt sie wohl als Samenfresserin an Erysimum.

Der, aus festem Gewebe bestehende und mit feinen Erdtheilchen vermengte Sack ist flach, oval, dunkelgrau und von filzigem Aussehen. Im Innern ist er mit weifser Seide ausgesponnen. Er erreicht eine Länge von $3 \frac{1}{2}$ Linien und hat im Durchmesser kaum 2 Linien.

Die Raupe von derselben Gröfse ist strohgelb, dick, walzenförmig; der kleine spitze Kopf glänzend dunkelbraun, das gleichfarbige Nackenschild ist hell getheilt, während die beiden folgenden Brustringe oben nur bräunlich gerandet erscheinen. Seitwärts von diesen stehen paarweise braune Borstenwärzchen, sowie einzelne helle Härchen, mit denen auch der gelbbraune After versehen ist. Die Krallen sind braungefleckt.

Zur Verwandlung pflegt das Thierchen sein Gehäuse mit we-

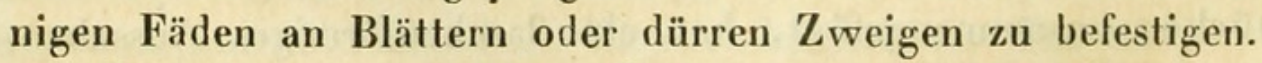

Die glänzend gelbbraune Puppe, mit den ihren Verwandten eigenthümlichen langen Fühlerscheiden, pflegt sich vor der Entwicklung bis zu zwei Drittheilen am Vorderende des Sackes herauszu. schieben. 
Die Motte erscheint sehr lokal; doch da, wo sie vorkommt, stets in kleinen Gesellschaften, Mitte Mai an den Blüthen des Knoblauchs-Hederich.

Um Frankfurt an der oberen Schweinsstiege.

\section{Adela Sulzeriella Zell.}

Unter Ligusterhecken überwintert die, von Herrn Mühlig entdeckte, einen Sack tragende Raupe. Ohne $Z_{\text {weifel lebt das Thier- }}$ chen in seiner Jugend in den Blüthen von Liguster und späler unter seinem Versteck von dürrem Laub und weichen Pflanzenabfällen u. s. w.

Der erdfarbene, $3 \frac{1}{2}$ Linie lange und in seinem Durchmesser über eine Linie breite Sack ist von länglicher Form und aus feinen Sandtheilchen zusammengesetzt; innen ist er mit weilser Seide ausgesponnen.

Das $2 \frac{1}{2}$ Linien lange Räupchen ist wulstig, nach hinten verdickt, glänzend schmutzigweifs, gegen den herzförmigen Kopf und gegen den After hin schwach gelbbraun; der erstere mit dem Nakkenschild und den beiden folgenden Brustringen glänzend dunkelbraun; doch auf letzteren ist diese Farbe mehr verwaschen, während das Nackenschild weifs gerandet ist.

Der Darmkanal stellenweise dunkel durchscheinend; die Krallen sind braun.

Die hellbraune Puppe tritt bei der Entwicklung ziemlich weit aus dem Sack hervor.

Die Motte fliegt einzeln in der Endhälfte Mai und Anfang Juni um Ligusterhecken.

Um Frankfurt bei Oberrad, am Gailsweg und in der Nähe der Kettenhöfe; bei Lorsch an der Bergstrafse.

\section{Nemotois Schiffermüllerellus S. V.}

Die sacktragende Raupe findet man schon im Spätherbst unter den Büschen von Ballota nigra, in deren Blüthen sie wahrscheinlich ihre Jugend verbringt. Später lebt sie ausschliefslich von den Blättern dieser Pflanze oder ihren Abfällen.

Der braune, filzige Sack ist abgerundet, in der Mitte verengt bis zu 2 Linien, während die beiden Enden an 5 Linien ausweisen. Sonst ist der Sack bei seinen flachen Rändern stark gewölbt.

Die Raupe ist dick, wulstig, weifslich mit glänzend schwarzem Kopf und Nackenschild; auf den Brustringen ist diese Farbe etwas schwächer, dagegen der After wieder schwarz; die Krallen sind 
braungefleckt. Zur Verwandlung wird der Sack mittelst einiger starker Fäden an naheliegende Erdtheilchen befestigt.

Die Puppe ist glänzend hellbraun.

Die Motle selten und lokal im Juni. Auf dem Mühlberg bei Frankfurt.

\section{Nemotois Dumerilellus Dup.}

Die Raupe entdeckte ich den 6. April 1860 unter den Blättern des Hypericum perforatum. Der von ihr bewohnte Sack ist $3 \frac{1}{2} \mathbf{L i}-$ nien lang und $1 \frac{1}{2}$ Linien breit, flach, länglich-oval, aus einem Geschiebe von Sandtheilchen bestehend, die dem Bau theilweise eine kreisförmige Zeichnung verleihen.

Die Raupe ist beinfarben.

Der herzförmige Kopf glänzend dunkelbraun; das Nackenschild fast schwarz, oben hell getheilt, seitlich mit einzelnen bellen Härchen versehen.

Die Brustringe sowie die Krallen sind braun.

Die Motte findet sich in der Endhälfte Juli gesellschaftlich an trockenen Sandstellen um die Futterpflanze der Raupe.

Auf der Bieberer Höhe bei Offenbach; neue Mörfelder Land. strafse bei Frankfurt und auf der Mombacher Haide bei Mainz.

\section{Gelechia Peliella Tr.}

Die sehr flüchtige Raupe entdeckte ich in Gesellschaft meines Freundes Stainton den 22. Mai 1859 an Rumex acetosella, woselbst sie nächst den Wurzelblättern in leichtem Gespinnste verborgen lebt. Sie ist 6 Linien lang und ist dunkelrothbraun.

Der Kopf und das Nackenschild sind glänzend schwarz, ebenso die Bauchfüfse. Einzelne kurze Härchen sind über den ganzen Körper zerstreut.

Die Verwandlung geschieht in einem dünnen Seidengewebe zwischen Moos oder dürrem Laub.

Die Motte ist nicht selten im Juni und Juli an Waldrändern. Sie sitzt gern an Baumstämmen.

Um Frankfurt am Hartweg und bei Griesheim; bei Königstein im Taunus und beim Mombach nächst Mainz.

Die Raupe ist in Entomologist's Annual 1860. p. 144 von Stainton kurz erwähnt. 
Gelechia Cauligenella A. Schmid nov. sp.

Capite thoraceque albis; alis anterioribus nigro-fuscis, fascia ante apicem interrupta, maculisque duabus subsuturalibus flavo-albidis. - Exp. alar. 5-6 Lin.

Vit G. Basultinella $\mathrm{Z}$ ell. verwandt. Kopf und Halsschild weifs, etwas ins Gelbliche fallend. Palpen gleichfarbig, das letzte schmälere Glied vor der Spitze gebräunt. Fühler kürzer als der Körper, schwarz, schmal hell geringelt. Vorderflügel schwarzbraun, mit hellerer Schuppenbeimischung. Vor dem Innenrand zwei gelblichweifse, mit dunkleren Schuppen gemischte, unregelmäfsige Flekken, wovon der eine vor der Flügelmitte, der andere in der Mitte steht und beide ein tiefschwarzes Fleckchen zwischen sich haben. Zuweilen erstrecken sich beide etwas bindenartig nach dem Vorderrand. Zwischen der Mitte und der Spitze des Flügels befindet sich eine durchgehende, schmale, gelblichweifse, in der Mitte etwas unterbrochene Binde. Die Franzen grau mit einer feinen, dunkeln Theilungslinie.

Die Hinterflügel nebst ihrem Franzen grau, nach der Spitze zu etwas dunkler.

Unterseite der Flügel grau, die helle Zeichnung kaum angedeutet. Hinterleib gelblichgrau. Schenkel, Schienen und Tarsen gelb. lich, nach Aufsen schwarz gefleckt.

Die Raupe fand ich 1860 im Juni in den Anschwellungen der Stengel von Silene nutans. Sie erreicht eine Länge von $2 \frac{1}{2}$ Linien. ist schmutziggelbgrün mit glänzend dunkelbraunem Kopf, nebst gleichfarbigem hellgetheiltem Nackenschild. Ueber den Rücken zieht sich eine gelbbraune verlosehene Längslinie. Das lebliafte Thierchen wird in der Erde gegen Ende Juni zur gelbbraunen Puppe.

Die Motte entwickelt sich Endhälfte Juli bis Mitte August. Im Freien noch nicht beobachtet.

Auf der Mombacher Haide bei Mainz.

\section{Gelechia Sequax Haw.}

Die Raupe fand ich in der leizten Maihälfte 1848 an Helianthemum vulgare zwischen den Blätlern der Pflanze und dem Stengel in röhrenartigen Gespinnsten. Sie ist 3 Linien lang und schmuzziggrün; der Kopf herzförmig, glänzend gelbbraun, ebenso das Nackenschild; beide schwarz gefleckt und, wie die Frefsspitzen, dunkel gerandet.

Der ganze Körper ist mit vielen schwarzen haartragenden Pünkt. 
chen versehen. Die Bauchseite ist von hellerer Färbung. Die Verwandlung geschieht in der Erde.

Die Motte im Juli in einzelnen Exemplaren auf kalkigem Hügelland.

Auf der Bieberer Höhe bei Offenbach.

\section{Sophronia Humerella S. V.}

Die Raupe fand ich zuerst am 27. Mai 1855 in den zusammengesponnenen Blättern der Artemisia campestris. Sie erreicht ausgewachsen eine Länge von 2 Linien. Sie ist hellgrün und hat drei dunkele Längslinien über den Rücken. Der kleine herzförmige Kopf ist glänzend gelbbraun, von gleicher, jedoch hellerer Färbung das dunkelgefleckte Nackenschild. Der Körper ist mit kaum sichtbaren hellen Härchen besetzt. Die Verwandlung findet statt auf der Erdoberfläche, unter Pflanzenabfällen.

Die Motte wurde im Juni stellenweise in geringer Anzahl in der Nähe der Futterpflanze der Raupe gefangen.

Auf der Mombacher Haide bei Mainz; auf der Bieberer Höhe bei Offenbach; in den Flörsheimer Steinbrüchen am Main; Eberstadt nächst Darmstadt.

\section{Acrolepia Cariosella Zeller.}

Das von Herrn Mühlig entdeckte Räupchen lebt Anfangs Juli in den Trieben von Gnaphalium sylvaticum. Es erreicht eine Länge von $2 \frac{1}{2}$ Linien und ist lebhaft gelb. In Folge seiner tiefen Ringeinschnitte erscheint es wulstig. Der kleine herzförmige Kopf ist hellbraun, dunkel gerandet; der Darmkanal schwach durchschimmernd. Krallen und Bauchfüfse von der Farbe des Körpers. Die Verwandlung geschieht in dem Filz der Unterseite der Blätter.

Die Motte findet sich Endhälfte Mai und dann wieder Ende Juli und Anfangs August auf lichten Waldstelien.

Fundorte: nächst Frankfurt bei Isenburg und am Heussenstammer Fahrweg.

\section{Elachista Chrysodesmella Zeller.}

Die Raupe entdeckte ich den 3. April 1859 in den Blättern von Brachypodium pinnatum in einer, von der Spitze an abwärts laufenden, sich bald stark ausdehnenden, bräunlichen Mine.

Die Raupe erreicht in ihrem vollendeten Wachsthum eine Länge von $2 \frac{1}{4}$ Linién. Sie ist grünlichgelb, glänzend, mit dunkeln Ringeinschnitten. Der Kopf ist gelbbraun, dunkel gerandet. Das Nak- 
kenschild heller, schwach getheilt; die Brustringe bräunlich begränzt. Der Darmkanal dunkel durchscheinend; seitlich sind kleine Grübchen bemerkbar; die Krallen dunkelbraun. - Die Verwandlung geschieht auf dem bewohnten Grasblatt, nächst dem Absatze des Stieles.

Die Puppe ist glänzend gelbbraun, doch ohne sonstige Zeichnung, und kantig.

Die Motte findet sich selten Ende Mai. Um Frankfurt am Hainerweg in verlassenen Kalksteinbrüchen.

\section{Pterophorus Loewii Zeller.}

Die Raupe lebt Ende Juli bis September an manchen Orten häufig an dem Tausendguldenkraut (Erythrea centaureum) gewöhnlich in den grünen Samenkapseln, welche sie leer frifst, versteckt. Der ausgestofsene hellbraune Koth bürgt für das Vorhandensein des Thierchens. In diesem Falle darf man nur einige Pflanzen einsammeln, um täglich eine Anzahl Raupen, welche zur Verwandlung die Kapseln verlassen, zu erhalten; selbst wenn bereits die ersten Geistchen erscheinen und Puppen jeden Alters vorhanden sind, verlassen noch Raupen die Kapseln. - Erwachsen erreicht die Raupe eine Länge von 4-5 Linien. Sie ist gelbgrün mit dunkler Rückenader und einer ebenso gefärbten, jedoch schwächeren Längslinie beiderseits. Die Ringeinschnitte sind tief und kurz, einzelne hellbraune Härchen sind überall sichtbar.

Der kleine Kopf ist schwarzgelb mit zwei schwarzen Pünktchen. Die Frefsspitzen sind braun und ebenso die Krallen, dagegen haben die Bauchfüfse die Farbe des Körpers.

Die Puppe ist glatt, schön saftgrün oder röthlich, vorn abgestumpft und zeigt vor dem helleren Scheitel einen carminrothen Streifen, der sich nach der Schwanzspitze zu allmälig verliert.

Das Geistchen findet sich Ende August und im September auf trocknen, doch pflanzenreichen Stellen und Waldrändern nicht selten.

Um Frankfurt an der Babenhäuser Landstrafse und im Ginheimerwäldchen; auf der Bieberer Höhe bei Offenbach.

In Sepp, Nederlandsche Insekten, einem in Deutschland wenig bekannten Werke, hat J. de Vries Th. 6. p. 177 tab. XLV. Fig. 1-8. die Naturgeschichte dieser Art geliefert. 


\section{Pterophorus Fuscus Retz.}

Die Raupe Mitte Mai in verschiedenen Gröfsen an Ehrenpreis (Veronica chamaedrys) frei auf der Pflanze. Sie erreicht eine Gröfse von 6 Linien, ist grasgrün, gegen den kleinen schwarzen Kopf etwas gelblicher gefärbt, mit starken Ringeinschnitten und einer dunkeln Rückenader. Seitlich ist sie merklich kantig, und an dieser Stelle geht die Grundfarbe in ein tieferes Grün über, während Krallen und Bauchfüfse eine bräunliche Färbung haben. Der ganze Körper ist mit langen hellen Härchen besetzt.

Die Puppe ist grün.

Der Schmetterling erscheint nach 14 Tagen und ist stellenweise häufig zur Endhälfte Juni an Waldrändern.

Um Frankfurt im Laubgesbusch und am Hartweg nach Schwanheim; Falkenstein im Taunus.

\section{Pterophorus Carphodactylus Hüb.}

Die Raupe findet sich erwachsen stellenweise in einiger Anzahl in den Herztrieben der Eberwurz (Conyza squarosa), woselbst auch die Verwandlung stattfindet. Sie erreicht eine Länge bis zu 4 Linien. Der Körper ist schmutzigweifs, glänzend, mit hellen feinen Härchen sparsam versehen. Der Kopf ist dunkelbraun; das Nackenschild fast schwarz, durch eine feine weifse Linie getheilt; auf jedem Leibesring stehen eilf längliche schwarze Querflecken. Die Krallen sind schwarz.

Die Puppe ist hellbraun, glatt, ohne jede Behaarung.

Das Geistchen findet sich Ende Mai und im Juni an Fahrwegen, in verIassenen Steinbrüchen, des Abends schwärmend.

Bei Frankfurt an der Darmstädter und Babenhäuser Landstrafse und auf dem Lerchesberg. 


\section{$2 \mathrm{BHL}$ Biodiversity Heritage Library}

Schmid, Anton. 1863. "Beiträge zur Naturgeschichte der Schmetterlinge." Berliner entomologische Zeitschrift / herausgegeben von dem Entomologischen Vereine in Berlin 7(142)57-66. https://doi.org/10.1002/mmnd.18630070106.

View This Item Online: https://www.biodiversitylibrary.org/item/36389

DOI: https://doi.org/10.1002/mmnd.18630070106

Permalink: https://www.biodiversitylibrary.org/partpdf/209965

\section{Holding Institution}

Smithsonian Libraries

\section{Sponsored by}

Smithsonian

\section{Copyright \& Reuse}

Copyright Status: Public domain. The BHL considers that this work is no longer under copyright protection.

This document was created from content at the Biodiversity Heritage Library, the world's largest open access digital library for biodiversity literature and archives. Visit BHL at https://www.biodiversitylibrary.org. 